\title{
Incorporation of Mindfulness Exercises to Reduce Anxiety During Urodynamic Testing: A Randomized Single-Blind Controlled Pilot Trial
}

\author{
Pansy Uberoi, MD, MPH, Anna Smitherman, PhD, James Aden, PhD, \\ Grace Park, MD, and Forrest Jellison, MD
}

\section{Introduction}

$\mathbf{U}$ RODYNAMICS (UDS) INVOLVES a study of the bladder in which the patient is accompanied by a health care team and involves placement of catheters in the bladder and/or other body cavities along with needles or probes, making the study quite invasive. Given such a setting, it is not surprising that patients experience physical and emotional distress. ${ }^{1,4-8}$ Mindfulness-based stress reduction (MBSR) exercises have shown a reduction in emotional and physical discomfort; therefore, the emotional and physical impact of short-term (MBSR-BE) breathing exercise were investigated before undergoing UDS. ${ }^{2,3}$

\section{Methods}

This a single-center investigator-blinded randomized parallel-group randomized control pilot trial is registered with ISRCTN registry-Trial No. NCT03625843 that adhered to consort criteria and was performed between August 2016 and December 2017. Eligibility criteria were age 18 years and participants were excluded if were pregnant or had neurogenic bladder. Subject randomization using permutated blocks and assignments was concealed in consecutively numbered opaque envelopes. Those in the MBSR-BE group participated in psychologist led mindfulness practice, all with the same psychologist who read the same script, which took $10 \mathrm{~min}$ to complete, whereas the control group patients were seated in a quiet empty room where they waited for $10 \mathrm{~min}$.

Control and MBSR-BE groups then underwent UDS by a single blinded provider who conducted the testing. Baseline, upon undergoing control or MBSR-BE, and after UDS, anxiety and pain levels were obtained. Anxiety was assessed with the state-trait anxiety inventory (STAI-6), pain was measured using a visual analog scale (VAS) for pain, and after completion of UDS, patients also completed a clinically validated UDS questionnaire that measured emotional and physical discomfort. ${ }^{1}$
Statistical analysis was performed using JMP version 12.3 Students' $t$-test was used to compare the continuous variables and VAS scores between groups. Ordinal data were compared with the data of Kruskal-Wallis test, and categorical data were assessed using chi-squared test. Paired $t$-test along with a repeated measures analysis of variance was used to compare the mindfulness versus control groups' STAI-6 and VAS over time. $p$-Values $<0.05$ were considered significant. A sample size calculation was performed to detect a clinically significant difference of the STAI-6 with accrual of 51 equally allocated in each arm with power of $80 \%$, alpha $5 \%$, and to allow for a $5 \%$ dropout. This is a pilot study that is underpowered, but designed to estimate effect and feasibility.

\section{Results}

A total of 27 patients were enrolled, 13 patients were randomized to the MBSR-BE arm and 14 to the control arm. Demographics, percentage of patients who had prior UDS testing, presenting symptoms, and anxiety and pain scores were similar between groups.

After UDS, anxiety was similar between groups (Fig. 1), as were pain scores. Fear was statistically lower in the mindfulness group, $p=0.04$. Patient-perceived physical discomfort after UDS was similar between groups. Emotional discomfort was ranked as worse than physical discomfort, by $15 \%$ of participants in the control group, this did not reach significance. Equal number of subjects in both groups reported the test as being as expected.

\section{Discussion}

In this study, mindfulness breathing exercises did not reduce anxiety or pain; however, there are some suggestions that it has an impact on the emotional experience during UDS. The MBSR-BE group reported significantly less fear than the control group. In addition, none of the patients in the MBSR-BE group reported their worst discomfort as emotional, whereas emotional discomfort was "the worst" 


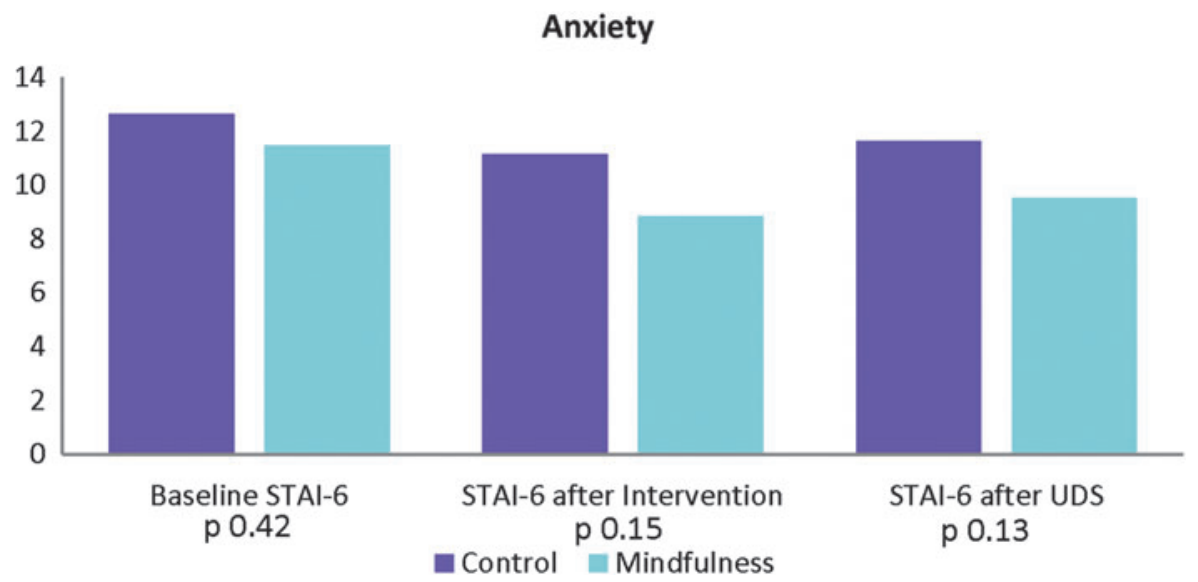

FIG. 1. STAI-6 scores of participants with corresponding $p$-values at baseline, after intervention or control, and after UDS in control and mindfulness groups. STAI-6, state-trait anxiety inventory; UDS, urodynamics. as perceived by $15 \%$ in the control group, which is similar to the findings reported by Suskind et al. to patients undergoing standard clinic UDS. ${ }^{1}$

This study is limited by confounding effect with several patients in the control arm providing unsolicited feedback stating that they engaged in short naps or prayer while waiting in the quiet empty room that is not typically experienced in the busy clinic waiting area. These behaviors may have created a "mindfulness effect" that might reduce anxiety or pain. As a pilot study, it is limited in number, thus, not powered to obtain significant differences.

\section{Author Disclosure Statement}

No competing financial interests exist.

\section{References}

1. Suskind AM, Clemens JQ. Patient perceptions of physical and emotional discomfort related to urodynamic testing: A questionnaire-based study in men and women with and without neurologic conditions. Urology 2015;85: 547-551.

2. Kanter G, Komesu YM, Qaedan F. Mindfulness-based stress reduction as a novel treatment for interstitial cystitis/bladder pain syndrome: A randomized controlled trial. Int Urogynecol J 2016;27:1705.
3. Erisman SM, Roemer L. A Preliminary investigation of the effects of experimentally-induced mindfulness on emotional responding to film clips. Emotion 2010;10:72-82.

4. Solomon ER, Ridgeway B. Interventions to decrease pain and anxiety in patients undergoing urodynamic testing: A randomized controlled trial. Neurourol Urodynam 2016;35:975-979.

5. Shim JS, Chae JY, Kang SG. Can listening to music decrease pain, anxiety, and stress during a urodynamic study? A randomized prospective trial focusing on gender differences. Urology 2017;104:59-63.

6. Yiou R, Audureau E, Loche C, et al. Comprehensive evaluation of embarrassment and pain associated with unvasive urodynamics. Neurol Urodynam 2015;34:156-160.

7. Yeung JY, Eschenbacher MA, Pauls RN. Pain and embarrassment associated with urodynamic testing in women. Int Urogynecol J 2015;25:645-650.

8. Shaw C, Williams K, Assassa PR, Jackson C. Patient satisfaction with urodynamics: A qualitative study. J Adv Nursing 2000;32:1356-1363.

Address correspondence to:

Forrest Jellison, MD Brooke Army Medical Center: Urology Clinic 3551 Roger Brooke Drive Fort Sam Houston, TX 78234

E-mail: fjellison@1lu.edu 